\title{
RETRACTED ARTICLE: Regularization of Divergent Integrals: An Approach Using Generalized Functions
}

\author{
V. V. Zozulya
}

Received: 13 March 2012 / Accepted: 29 August 2013 /

Published online: 27 September 2013

(C) Springer Science+Business Media New York 2013

This article has been retracted by Springer and the Editor-in-Chief of Advances in Computational Mathematics. Due to a technical mishap, a version of the manuscript was published that was not the finally accepted manuscript. Therefore, the correct accepted version has been published as a new article (DOI 10.1007/s10444-014-9399-3). We apologize sincerely for the confusion caused. 